\title{
Direct determination of impurities in high purity silicon carbide by inductively coupled plasma optical emission spectrometry using slurry nebulization technique
}

\author{
Zheng Wang ${ }^{\mathrm{a}, \mathrm{b}}$, Deren $\mathrm{Qiu}^{\mathrm{c}}$, Zheming Ni ${ }^{\mathrm{d}}$, Guangyi Tao ${ }^{\mathrm{a}}$, Pengyuan Yang ${ }^{\mathrm{c}, *}$ \\ a Shanghai Institute of Ceramics, Chinese Academy of Sciences, Shanghai 200050, China \\ ${ }^{\mathrm{b}}$ Graduate School of Chinese Academy of Sciences, Beijing 100039, China \\ ${ }^{\mathrm{c}}$ Department of Chemistry, Fudan University, Shanghai 200433, China \\ ${ }^{\mathrm{d}}$ Research Center for Eco-Environmental Sciences, Academia Sinica, P.O. Box 2871, Beijing 100085, China
}

Received 29 March 2006; received in revised form 16 June 2006; accepted 17 June 2006

Available online 27 June 2006

\begin{abstract}
A novel method for the determination of $\mathrm{Al}, \mathrm{Ca}, \mathrm{Cr}, \mathrm{Cu}, \mathrm{Fe}, \mathrm{Mg}, \mathrm{Mn}, \mathrm{Ni}$ and $\mathrm{Ti}$ in high purity silicon carbide (SiC) using slurry introduction axial viewed inductively coupled plasma optical emission spectrometry (ICP-OES) was described. The various sizes of SiC slurry were dispersed by adding dispersant polyethylene imine (PEI). The stability of slurry was characterized by zeta potential measurement, SEM observation and signal stability testing. The optimal concentration of PEI was found to be $0.5 \mathrm{wt} \%$ for the SiC slurry. Analytical results of sub- $\mu \mathrm{m}$ size SiC by the slurry introduction were in good accordance with those by the alkaline fusion method which verified that determination could be calibrated by aqueous standards. For $\mu \mathrm{m}$ size SiC, results of most elements have a negative deviation and should be calibrated by the Certified Reference Material slurry. Owing to a rather low contamination in the sample preparation and stability of the slurry, the limits of detection (LODs), which are in the range of 40-2000 $\mathrm{ng} \mathrm{g}^{-1}$, superior to those of the conventional nebulization technique by ICP-OES or ICP-MS.
\end{abstract}

(C) 2006 Elsevier B.V. All rights reserved.

Keywords: Silicon carbide; Slurry introduction; Inductively coupled plasma optical emission spectrometry; Polyethylene imine

\section{Introduction}

Powdery silicon carbide ( $\mathrm{SiC}$ ) has increasingly been used as one of the basic materials for the production of advanced ceramics. Due to its high thermal strength and refractory character, $\mathrm{SiC}$ has been widely used in a number of industrial fields such as heat engine, gas turbines and high temperature energy conversion system, and nuclear and fusion reactors $[1,2]$. However, its properties are significantly affected by the presence of impurities, even at very low levels of concentration. Determination of impurities in SiC powders is, therefore, of great importance.

Analytical methods with multielement ability such as optical emission spectrometry or mass spectrometry with inductively coupled plasma (ICP-OES or ICP-MS) should be applied.

\footnotetext{
* Corresponding author. Tel.: +86 21 65642009; fax: +86 2165642009

E-mail addresses: wangzheng@mail.sic.ac.cn (Z. Wang), pyyang@fudan.edu.cn (P. Yang).
}

Routine analysis of ICP spectrometry demands the sample in form of solution. However, it is chemically difficult to bring SiC sample into solution. Broekaert and co-workers [3-5] have reported that $\mathrm{SiC}$ could be decomposed with a mixture of $\mathrm{HNO}_{3}+\mathrm{H}_{2} \mathrm{SO}_{4}+\mathrm{HF}$ in a PTFE bomb. But the power of detection was poor for the high blanks relating to the use of fuming $\mathrm{H}_{2} \mathrm{SO}_{4}$. The decomposition of $\mathrm{SiC}$ by fusion with $\mathrm{Na}_{2} \mathrm{CO}_{3}+\mathrm{Na}_{2} \mathrm{~B}_{4} \mathrm{O}_{7}$ or $\mathrm{Li}_{2} \mathrm{~B}_{4} \mathrm{O}_{7}$ has been proved efficient [6]. However, blank of flux and high salt content considerably limit its application, especially for the samples in high purity. In order to avoid the problems causing from dissolution, direct solid analysis of ceramics by ICP-OES using solid sample introduction techniques have been reported over the last years [3,4,7-9]. With the use of electrothermal vaporization (ETV)-ICP-OES [8], the lower limits of detection could be achieved. However, the analyte/matrix separation in the vaporizer is not complete and as a consequence, the different behavior of $\mathrm{Cu}$ and $\mathrm{Fe}$ when processed from aqueous standards and from silicon carbide in the presence of Freon, calibration using aqueous standard solu- 
tion is not sufficiently accurate. With the application of the tungsten coil ETV-ICP-OES using slurry introduction [9], the sample mass is limited to $40-60 \mu \mathrm{g}$, which require high degree of sample homogeneity; element Ti has poor accuracy even using standard additions technique. For the slurry nebulization ICP-OES, it is simple to implement, is inexpensive and requires little instrument modification. To prepare a stable and homogeneous aqueous slurry, addition of a dispersing agent is generally recommended $[10,11]$. Analysis of SiC powder by slurry introduction ICP-OES was reported by Broekaert and co-workers $[3,4]$. However, due to the particle size distribution of the powders and stability of the slurry have not been investigated in detail, large deviation, especially for $\mathrm{Fe}$, from the results of the other procedures occurs. All above-mentioned methods only analyze impurities in submicrometer level fine $\mathrm{SiC}$ powder.

ICP-OES is still a most appropriate technique for elemental analysis [12], especially for those light elements, which are very important for advanced ceramics [7]. It is also noticeable that slurry introduction technique for analytical atomic spectrometry has been used for the analysis of many advanced materials, but details of various slurry preparation and characterization are still very scarce [13]. On the other hand, one should be cautious when slurry nebulization ICP-OES technique was used to analyze ceramic powders because the transport and nebulization efficiency are strongly influenced by particle size, the stability and homogeneity of the aqueous slurries $[11,14,15]$.

In this work, various particle sizes sample from fine- to coarse-grained SiC powders were selected. The stable and homogeneous suspensions were prepared and characterized with various methods. The prepared slurries were nebulized and carried into axially viewed ICP torch for the determination of impurities in SiC. The simple aqueous standards and Certified Reference Material as calibration methods were used. The results are presented and discussed.

\section{Experimental}

\subsection{Instrumentation and operating conditions}

All determinations were performed on a VISTA AX ICPOES spectrometer with axial viewed configuration (Varian, Palo Alto, CA, USA). The sample introduction system consists of a V-groove nebulizer and a reduced-volume Sturman-Masters Type spray chamber made of poly-tetrafluorethylene (PTFE). The operating parameters and selected analytical lines are listed in Table 1.

\subsection{Reagents and materials}

All reagents were of guarantee grade (first-rate reagent). The solutions were prepared with Milli-Q water $(18 \mathrm{M} \Omega \mathrm{cm})$. The multielement working standards were prepared from $1000 \mathrm{mg} \mathrm{L}^{-1}$ aqueous standards (Shanghai Institute of Measurement and Testing Technology, Shanghai, China). The blank and series standards were prepared containing same amount of dispersant.
Table 1

ICP-OES operating conditions and analytical lines

\begin{tabular}{ll}
\hline Viewing & Axial \\
\hline Injector tube diameter & $2.3 \mathrm{~mm}$ \\
Power & $1.25 \mathrm{~kW}$ \\
Nebulizer gas flow & $0.65 \mathrm{~L} \mathrm{~min}^{-1}$ \\
Sample uptake rate & $0.80 \mathrm{~mL} \mathrm{~min}^{-1}$ \\
& \\
Analytical lines & \\
\hline Element & Spectral line $(\mathrm{nm})$ \\
\hline $\mathrm{Al}$ & 308.215 \\
$\mathrm{Ca}$ & 317.933 \\
$\mathrm{Cr}$ & 283.563 \\
$\mathrm{Cu}$ & 324.754 \\
$\mathrm{Fe}$ & 238.204 \\
$\mathrm{Si}$ & 251.432 \\
$\mathrm{Mg}$ & 285.213 \\
$\mathrm{Mn}$ & 257.610 \\
$\mathrm{Ni}$ & 216.556 \\
$\mathrm{Ti}$ & 334.941 \\
$\mathrm{~V}$ & 311.837 \\
\hline
\end{tabular}

Various particle sizes of SiC samples were selected for testing. The sub- $\mu \mathrm{m}$ particles SiC (FCP 15, Norton Corp., Germany) (denoted as SiC-I) were directly utilized without grinding. The others, W10 and W40 (Shandong Qingzhou Micropowder Corp., China) and BAM-S003 (Federal Institute for Materials Research and Testing, Germany) (denoted as SiC-II, SiC-III and $\mathrm{SiC}-\mathrm{IV}$, respectively) were grinded in the lab by a vibration mill for $2 \mathrm{~min}$. The grinded samples were oven-dried at $110^{\circ} \mathrm{C}$ for $2 \mathrm{~h}$ then stored in a desiccator.

\subsection{Sample preparation}

Slurry was prepared by weighing the SiC sample then transferred into a $100 \mathrm{~mL}$ volumetric flask containing PEI (BDH Laboratory, UK) (0.5 wt\% PEI).

Sample was alternatively prepared by mixing the sample $(0.1 \mathrm{~g})$ with an alkaline flux $\left(2 \mathrm{~g}, \mathrm{Na}_{2} \mathrm{CO}_{3}+\mathrm{Na}_{2} \mathrm{~B}_{4} \mathrm{O}_{7}\right)$ and fused in a $\mathrm{Pt}$ crucible for $30 \mathrm{~min}$. After cooling to room temperature, water was added to cover the fusion cake and left it overnight. The dissolved sample solution was transferred into a polyethylene volume flask and made up to the volume of $100 \mathrm{~mL}$ with water. The fusion prepared sample solution was used for the comparison to the slurry samples in the analysis.

\subsection{Characterization of slurries}

\subsubsection{Zeta potential measurement}

Zeta potential of the slurry was measured by a Zeta-Plus Analyzer (Brookhaven Instruments, USA).

\subsubsection{SEM observation}

The slurry was allowed to settle on a copper slice under gravity and a thin sediment film was formed. The sample sediment was then dried in the air and observed with a SEM (JSM-6700F, 
Japan). To obtain high-quality micrograph, the sediment was coated with a thin gold film prior to the observation.

\subsubsection{Particle size determination}

Particle size of the $\mu \mathrm{m}$ and sub- $\mu \mathrm{m}$ SiC in slurry was determined by a SICAS-4800 Photo-Sedimentometry (Shanghai Institute of Ceramics, CAS).

\section{Results and discussions}

\subsection{Grinding method and particle size distribution}

$\mathrm{SiC}$ has extreme hardness, abrasion resistance and is difficult to be ground. The original average diameter of SiC-II-SiC-IV powders were 8.56, 27.92, $9.2 \mu \mathrm{m}$, respectively. Two methods were used to grind the particles. The bottle and bead method was first used. $1.0 \mathrm{~g}$ sample was weighted and put into an $80 \mathrm{~cm}^{3}$ polypropylene bottle and $10 \mathrm{~g}$ of vitrified $\mathrm{Si}_{3} \mathrm{~N}_{4}$ beads was added. Dispersant solution was added up to the beads to be inundated, and the bottle was then shaken on a wrist-action laboratory flask shaker for $24 \mathrm{~h}$. The finely ground slurry was then transferred to a volumetric flask and diluted to the volume with additional dispersant and water. The blank has undergone a procedure same as the slurry and determined by ICP-OES. Little contamination was introduced because the high purity grinding material was used. However, the mill efficiency was not well, such as for the SiC-IV sample, after grinding, 90\% particle size of powder was larger than $5 \mu \mathrm{m}$. The average diameter was only a little decreased to $7.9 \mu \mathrm{m}$. The vibration mill method was then used. The solid was contained in the shell, together with the beads for grinding, and vibrated. The grinding material and particles collide with each other and the shell, resulting in the breakdown of solid particles. Contamination of tungsten, cobalt and few zirconium might be caused, but grinding was high efficient. Only few minutes of grinding time was needed. The particle size was decreased quickly. After grinding $2 \mathrm{~min}, 90 \%$ particle sizes of the SiC-II-SiC-IV powders were minimized to less than $5 \mu \mathrm{m}$. The average diameter was decreased to about $1.5 \mu \mathrm{m}$. The rigorous studies reported that slurry particles larger than $5 \mu \mathrm{m}$ (in some reports $2 \mu \mathrm{m}$ ) do not reach the plasma and result in a loss of signal [16]. Therefore, vibration mill method was used for all grind operations. The particle sizes of SiC-I were gained from the SEM image, as shown in Fig. 1. The particle size distributions of the original SiC-II-SiC-IV and the milled SiC-II-SiC-IV were shown in Figs. 2 and 3, respectively.

\subsection{Influence of $p H$ on the slurry stability and effect of dispersant}

The atomization efficiency of the slurry would be improved with the decrease of the particle size. It is known that fine particles in slurry are highly charged. The agglomerated particles have static charge originating from intrinsic charged groups or from adsorption of charged molecular or groups coexisting in the medium, such as $\mathrm{H}^{+} / \mathrm{OH}^{-}$. At isoelectric point (IEP), slurry will be unstable and the particles will be agglomerated. The slurry is stable only in case the zeta potential apart far from the IEP.
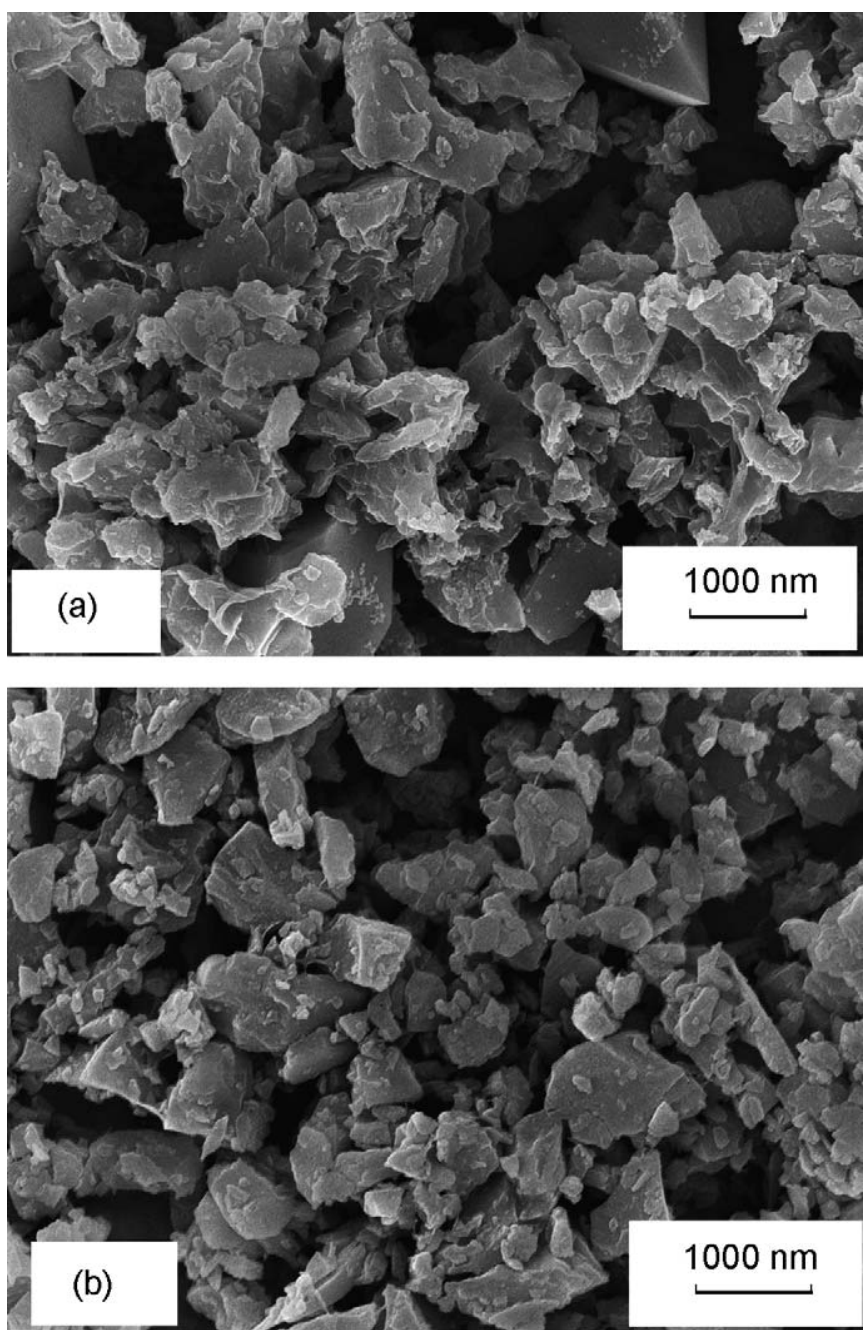

Fig. 1. SEM micrograph of sediments resulting from $2 \mathrm{wt} \%$ SiC-I suspension at $\mathrm{pH} 4.0$ : (a) without dispersants $(\times 15,000)$; (b) with $0.5 \mathrm{wt} \%$ PEI dispersants $(\times 15,000)$.

Fig. 4 shows the relationship between the zeta potential of $\mathrm{SiC}$ slurry and $\mathrm{pH}$. The IEP of the native sub- $\mu \mathrm{m}$ particle size $\mathrm{SiC}$ is about 2.8, similar to the value reported by Sun and Gao [17] Slurry would be stable in alkaline media for sub- $\mu \mathrm{m}$ particle size $\mathrm{SiC}$ because of the zeta potential apart far from the IEP, just as illustrated in Fig. 4. However, the viscosity became very high in basic medium, resulting in a great difference in aspiration as compared to the aqueous standards [15].

Effect of different concentrations of PEI on the zeta potential at different $\mathrm{pH}$ is also shown in Fig. 4. The zeta potentials kept constant in range of $\mathrm{pH} 2-8$. From Fig. 4 it can also be seen that about $0.5 \mathrm{wt} \%$ PEI may be the appropriate amount for dispersing SiC. Nevertheless, the concentration of the dispersant should be controlled because the excessive dispersant would destroy the stability of slurry and cause the increase of viscosity [15].

\subsection{Dispersion effect and stability of spectral line intensity}

The dispersion effect could also be illustrated by comparing the SEM micrographs of sediments resulting from $2 \mathrm{wt} \%$ 

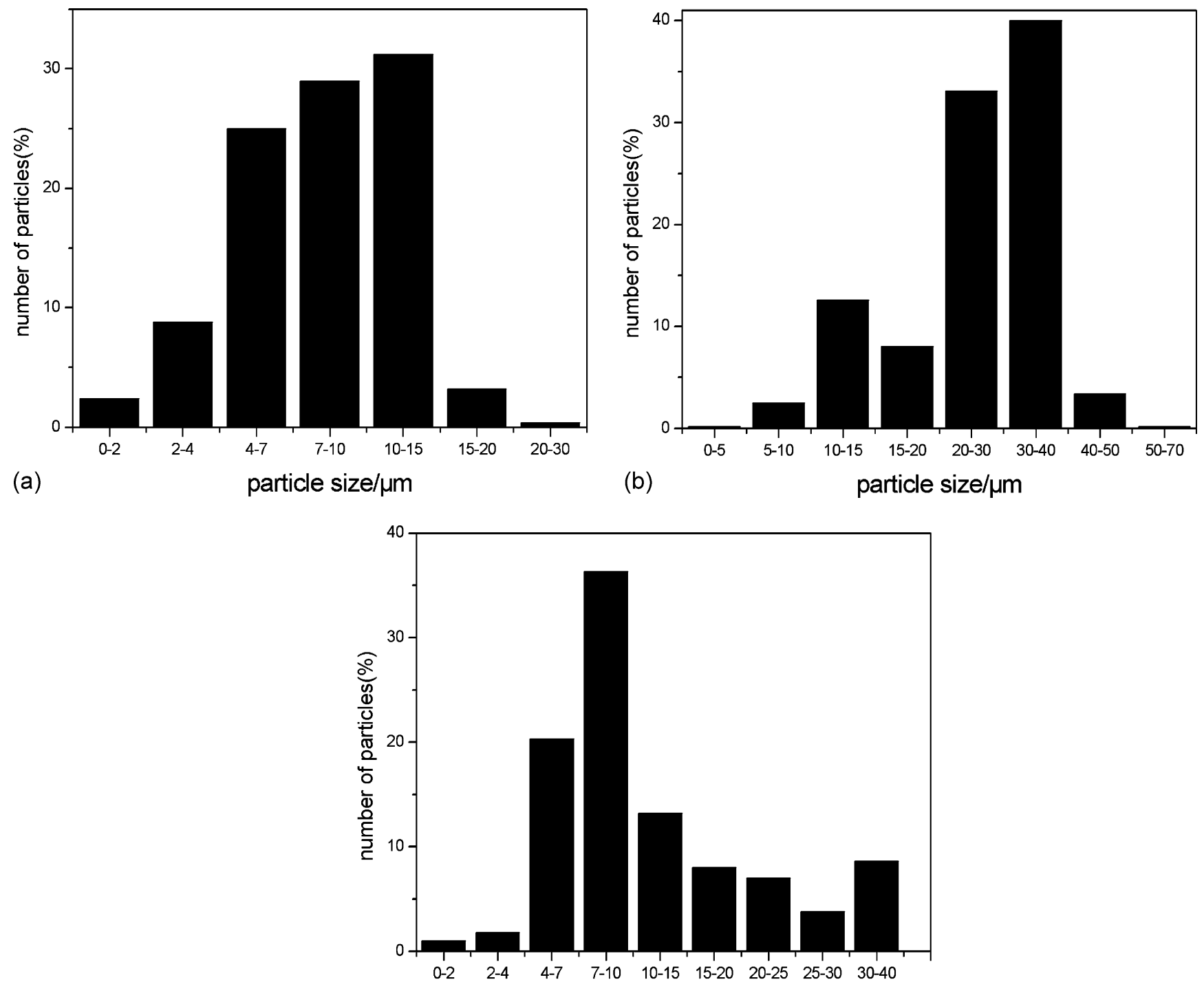

(c)

particle size/ $\mu \mathrm{m}$

Fig. 2. Bar diagram for original particle size distribution: (a) SiC-II; (b) SiC-III; (c) SiC-IV.

suspension without dispersant (Fig. 1(a)) and with $0.5 \mathrm{wt} \%$ PEI (Fig. 1(b)). Large flocks appeared in the sediments of suspensions in case no dispersant was added (Fig. 1(a)). Nevertheless, particles of the sediments with $0.5 \mathrm{wt} \%$ PEI were not highly agglomerated, verifying that particles were well dispersed in the suspension (Fig. 1(b)).

Slurry stability was experimentally tested as shown in Fig. 5. The nm size SiC slurry with no dispersant at IEP (zeta potential is zero) displayed unstable property. The normalized line intensities of matrix $\mathrm{Si}$ and impurities $\mathrm{Al}, \mathrm{Fe}$ were obviously decreased with time, as shown in Fig. 5(a). Comparatively, the slurry with dispersant PEI (2.0 wt\%), zeta potential of which was far from IEP, displayed the relatively stable intensities (Fig. 5(b)).

\subsection{Calibration}

Satisfactory result can be obtained from fusion prepared sample. Alkaline fusion needs high purity of the fusion reagents.
However, some trace elements are still too low to be determined due to the dilution. Using slurry introduction the reagent contamination and dilution can be avoided, so that trace elements can thus be determined. For sub- $\mu \mathrm{m}$ particle size of $\mathrm{SiC}$, the determined results by the slurry introduction method were in good accordance with those by the alkaline fusion method, verifying that calibration curves could be established by aqueous standards.

However, for the $\mu \mathrm{m}$ particle size $\mathrm{SiC}$, results by the slurry method have great deviation from the alkaline fusion results. Method of standard additions was verified no avail to the calibration. This might be predominantly attributed to the incomplete evaporation of the $\mu \mathrm{m}$ particle size $\mathrm{SiC}$ (high melting point $2600{ }^{\circ} \mathrm{C}$ ) and also to the different efficiency of transportation between $\mu \mathrm{m}$ particle size $\mathrm{SiC}$ suspension and aerosol of the aqueous solution [18]. In case that the slurry is introduced into the plasma and aqueous standards are used for calibration, different behaviors in transportation, evaporation and atomization will be occurred, unless the particle size is small enough and 


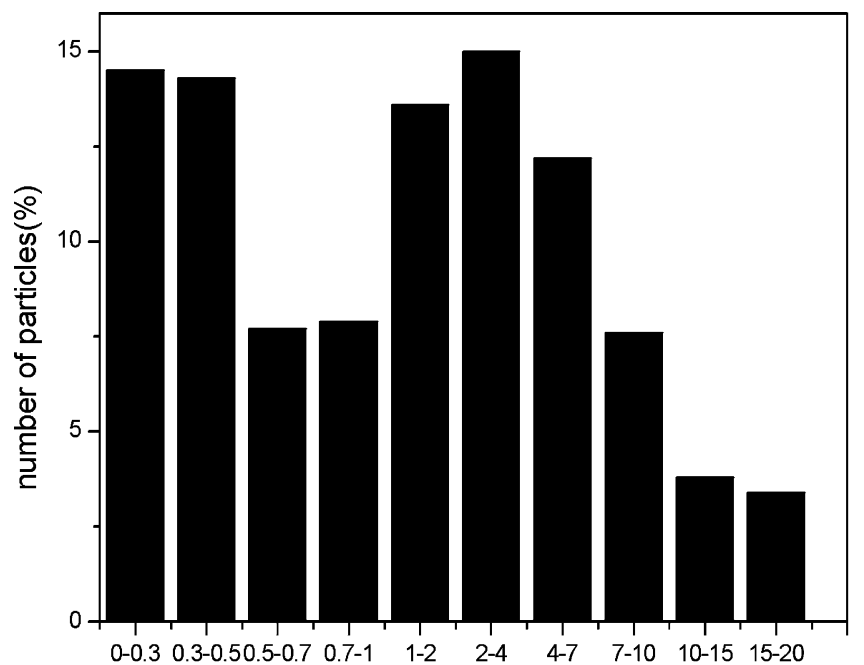

(a)

particle size/ $\mu \mathrm{m}$

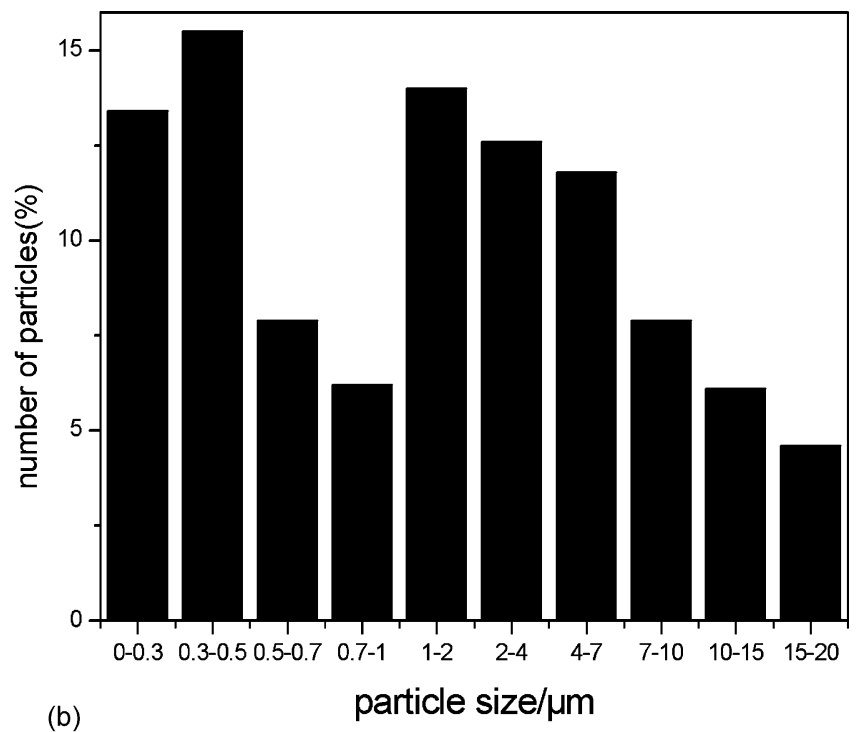

(b)

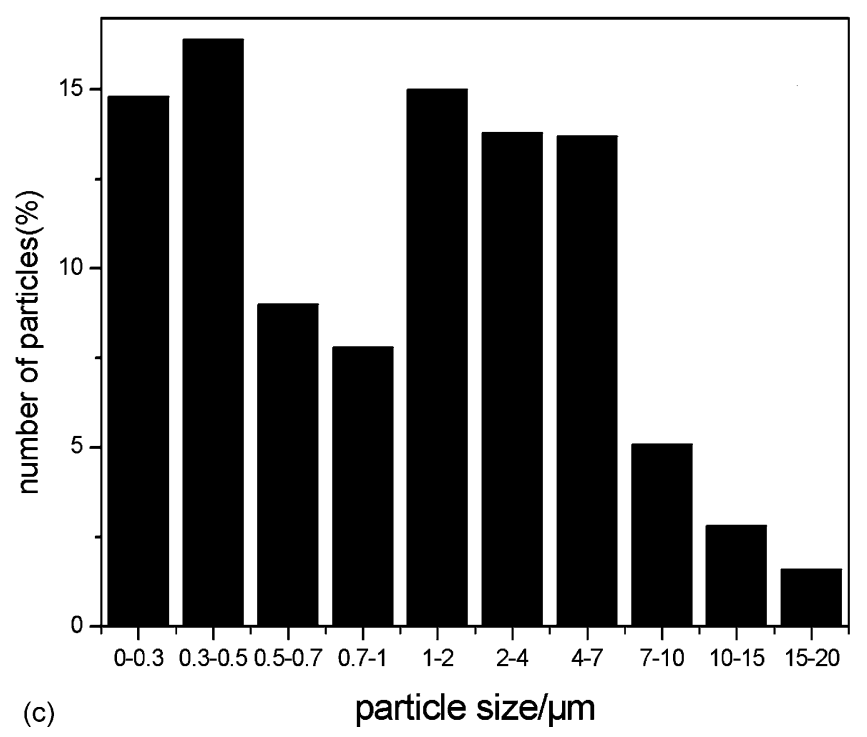

Fig. 3. Bar diagram for milled particle size distribution: (a) SiC-II; (b) SiC-III; (c) SiC-IV.

all the courses are carried out completely, just as in the case of sub- $\mu \mathrm{m}$ particle size of $\mathrm{SiC}$.

As has been mentioned, $\mathrm{SiC}$ particle size was not easily decreased. The original $\mathrm{SiC}$ particle sizes have great difference. But after the SiC powders were milled, the particle size distribution of SiC-II and SiC-III were approximately identical to that of SiC-IV. Thus, the deviation during transportation and incomplete evaporation could be corrected using the Certificate Reference Material SiC slurry which have been used as an alternative to aqueous standards in some analyses of solid sample [16]. Slurries of $0.1 \%, 0.25 \%$ and $1 \%\left(\mathrm{~m} \mathrm{~V}^{-1}\right)$ were prepared from SiC-IV sample and spectral line intensities were recorded under the optimum plasma operating conditions as listed in Table 1. Slurries for calibration were prepared using the same procedures as for the samples, containing the same amount of PEI. Results obtained shown that they were in good accordance with those by the alkaline fusion method (Table 4).

\subsection{Limits of detection (LODs)}

The LODs expressed in terms of three times of standard deviation (S.D.) of the blanks obtained from a high purity SiC slurry are given in Table 2. The LODs are compared with those obtained by the conventional nebulization ICP-OES [19]. The superiority of the slurry nebulization technique is evident: due to the quality control of the water and dispersant during the preparation of the slurry, low limits of detection, except $\mathrm{Mg}$, for elements of $\mathrm{Ca}, \mathrm{Mn}$, Ti and $\mathrm{V}$ being 6-15 times lower than those of the solution method. For the elements $\mathrm{Ni}, \mathrm{Cr}$ and $\mathrm{Fe}$ two to four times improvement of the limits of detection was achieved. The remarkable potential is found by comparing the method of ICP-MS [19]. It is, in most cases, better than the method of W-coil-ETV-ICP-OES [9], SIS-ETAAS [20] and INAA [19]. In these cases, a rather low contamination was introduced in the sample preparation and has good S.D. values for stable suspension. Comparatively, SoS-ETV-ICP-OES [8] were consistently 


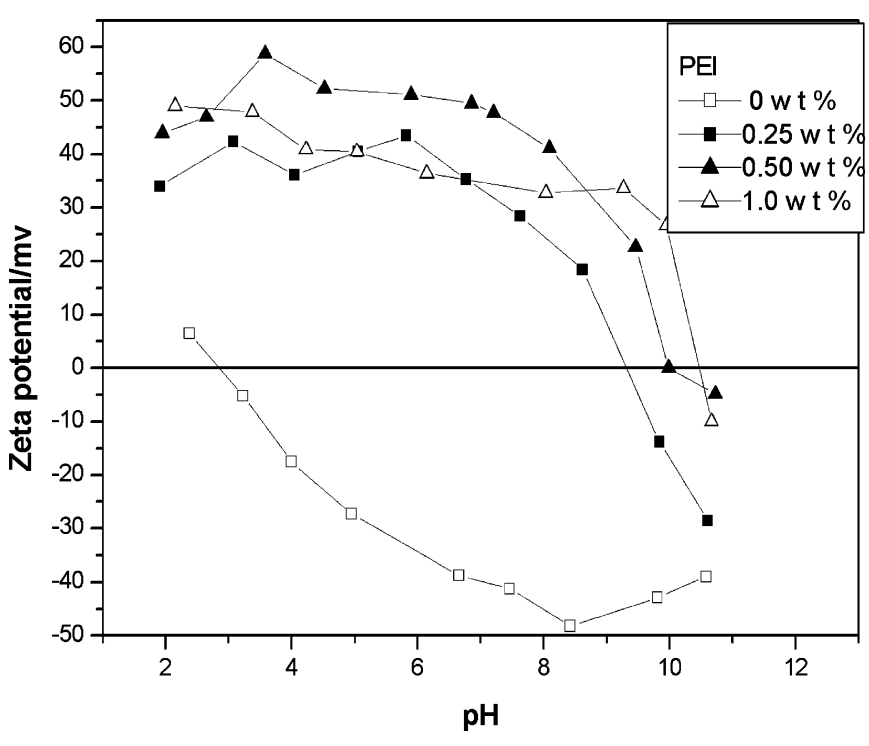

Fig. 4. Profiles of zeta potential vs. pH for SiC-I slurry at different PEI concen-

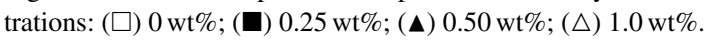

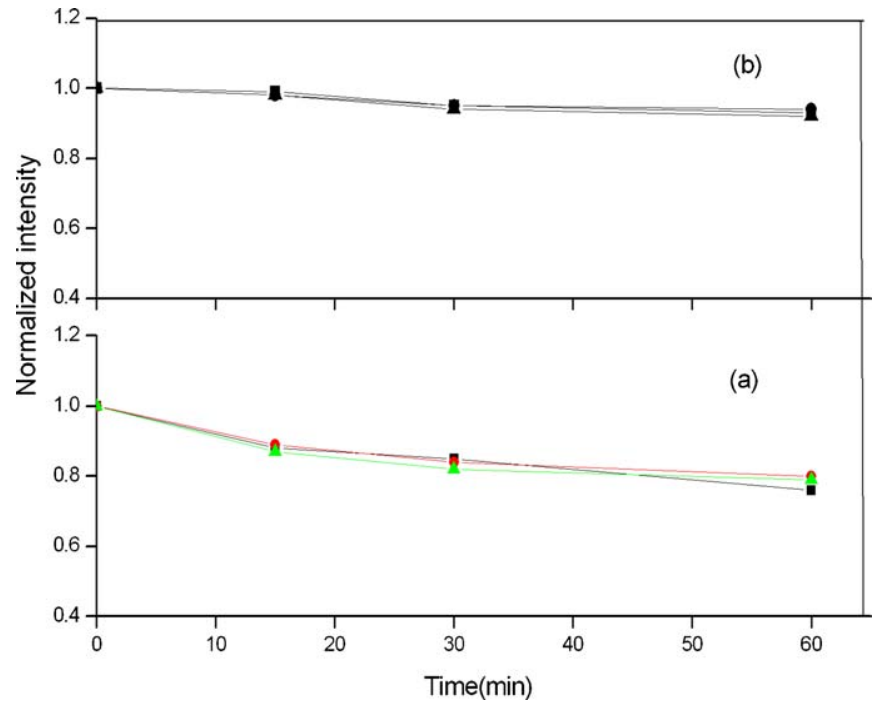

Fig. 5. Profiles of normalized line intensity vs. time for SiC-I slurry at $\mathrm{pH}$ 6.0:

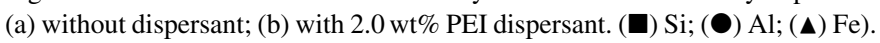

Table 2

Limits of detection for high purity $\mathrm{SiC}$ (for sample concentration of $1 \%, \mathrm{~m} \mathrm{~V}^{-1}$ ) using slurry ICP-OES and comparison to those reported by the other methods

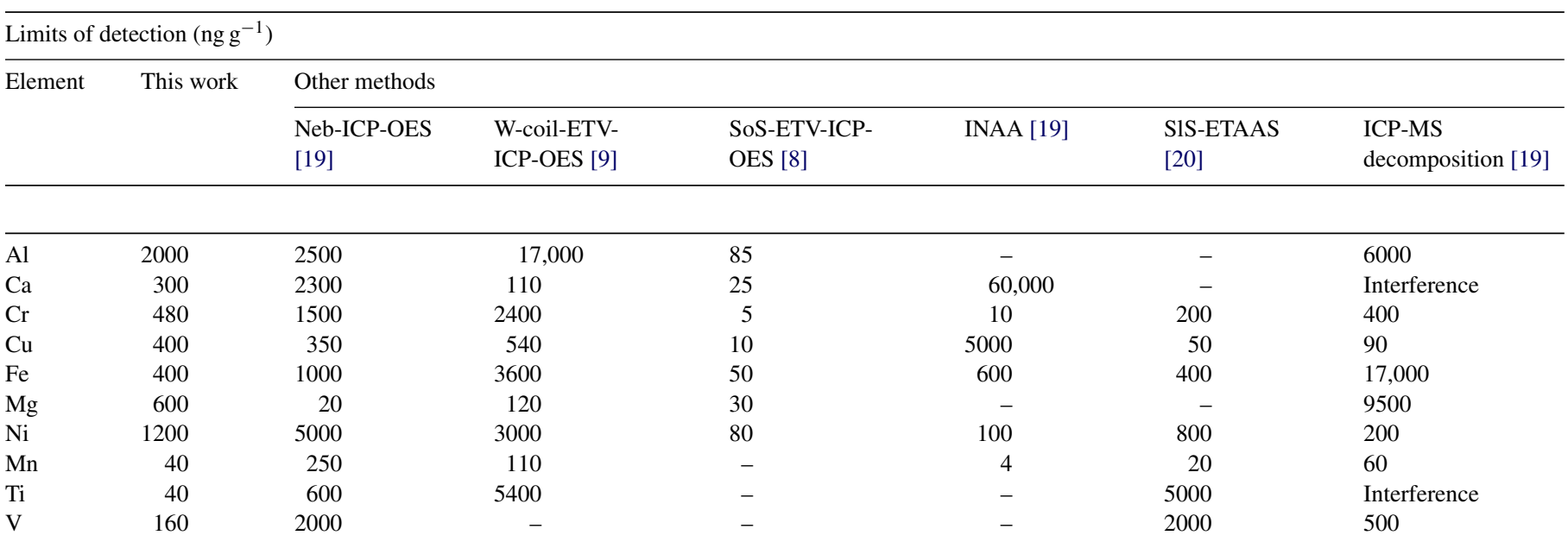

Note: Neb, nebulization; W-coil-ETV, tungsten electrothermal vaporization; SoS-ETV, solid sampling electrothermal vaporization; INAA, instrument neutron activation analysis; SIS-ETAAS, slurry sample introduction electrothermal atomic absorption spectrometry. (-), LOD not reported.

Table 3

Comparison of analytical results $\left(\mu \mathrm{gg}^{-1}\right)$ for $\mathrm{SiC}$, determined by alkaline fusion method to the slurry introduction, calibrated with aqueous standards and the certificate values of Certified Reference Material

\begin{tabular}{|c|c|c|c|c|c|}
\hline \multirow[t]{2}{*}{ Element } & \multicolumn{2}{|l|}{ SiC-I } & \multicolumn{3}{|l|}{ SiC-IV } \\
\hline & Alkaline fusion & Slurry introduction method & Alkaline fusion & Slurry introduction method & Certificate values \\
\hline Al & $248 \pm 18$ & $244 \pm 25$ & $373 \pm 18$ & $129 \pm 25$ & $372 \pm 20$ \\
\hline $\mathrm{Ca}$ & $29.4 \pm 2.1$ & $30.0 \pm 4.0$ & $27.5 \pm 2.0$ & $34.0 \pm 2.9$ & $29.4 \pm 1.8$ \\
\hline $\mathrm{Cr}$ & $1.5 \pm 0.3$ & $1.7 \pm 0.4$ & $3.5 \pm 0.6$ & $3.8 \pm 0.6$ & $3.5 \pm 0.4$ \\
\hline $\mathrm{Cu}$ & - & $0.18 \pm 0.06$ & $1.7 \pm 0.4$ & $1.4 \pm 0.8$ & $1.5 \pm 0.4$ \\
\hline $\mathrm{Fe}$ & $134 \pm 8$ & $118 \pm 10$ & $156 \pm 11$ & $81 \pm 16$ & $149 \pm 10$ \\
\hline $\mathrm{Mg}$ & $3.3 \pm 0.4$ & $3.4 \pm 0.6$ & $8.0 \pm 0.5$ & $11.6 \pm 0.8$ & $6.3 \pm 0.6$ \\
\hline $\mathrm{Mn}$ & - & $0.52 \pm 0.10$ & $1.47 \pm 0.21$ & $1.55 \pm 0.26$ & $1.44 \pm 0.17$ \\
\hline $\mathrm{Ni}$ & $10.1 \pm 1.6$ & $10.5 \pm 2.4$ & $34.2 \pm 2.0$ & $31.7 \pm 3.2$ & $32.9 \pm 2.7$ \\
\hline $\mathrm{Ti}$ & $88 \pm 5$ & $82 \pm 7$ & $82 \pm 5$ & $50 \pm 7$ & $79 \pm 4$ \\
\hline V & $24.6 \pm 2.1$ & $21.4 \pm 3.5$ & $41.9 \pm 2.5$ & $18.2 \pm 2.0$ & $41.4 \pm 2.8$ \\
\hline
\end{tabular}

Note: Certificate value $=$ mean value \pm 2 S.D., the others, mean value \pm 3 S.D $(n=5)$. 
Table 4

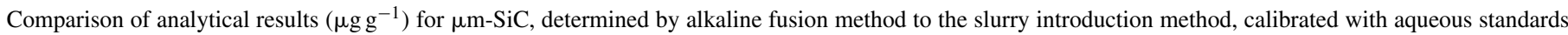
(I) and calibrated with the slurry prepared with the Certified Reference Material (II)

\begin{tabular}{|c|c|c|c|c|c|c|}
\hline \multirow[t]{2}{*}{ Element } & \multicolumn{3}{|l|}{ SiC-II } & \multicolumn{3}{|l|}{ SiC-III } \\
\hline & $\begin{array}{l}\text { Alkaline } \\
\text { fusion }\end{array}$ & $\begin{array}{l}\text { slurry introduction } \\
\text { method (I) }\end{array}$ & $\begin{array}{l}\text { slurry introduction } \\
\text { method (II) }\end{array}$ & $\begin{array}{l}\text { Alkaline } \\
\text { fusion }\end{array}$ & $\begin{array}{l}\text { Slurry introduction } \\
\text { method (I) }\end{array}$ & $\begin{array}{l}\text { Slurry introduction } \\
\text { method (II) }\end{array}$ \\
\hline $\mathrm{Al}$ & $142 \pm 19$ & $94 \pm 15$ & $150 \pm 25$ & $2040 \pm 200$ & $647 \pm 52$ & $1760 \pm 170$ \\
\hline $\mathrm{Ca}$ & $113 \pm 16$ & $108 \pm 15$ & $115 \pm 19$ & $138 \pm 12$ & $129 \pm 12$ & $135 \pm 15$ \\
\hline $\mathrm{Cr}$ & $5.5 \pm 0.6$ & $6.4 \pm 0.8$ & $6.5 \pm 0.8$ & $8.0 \pm 0.5$ & $8.3 \pm 0.9$ & $8.9 \pm 1.0$ \\
\hline $\mathrm{Cu}$ & $6.6 \pm 0.5$ & $5.4 \pm 0.6$ & $6.0 \pm 0.5$ & $5.3 \pm 0.6$ & $4.8 \pm 0.5$ & $5.0 \pm 0.8$ \\
\hline $\mathrm{Fe}$ & $1800 \pm 210$ & $703 \pm 65$ & $1600 \pm 240$ & $852 \pm 72$ & $402 \pm 42$ & $815 \pm 76$ \\
\hline $\mathrm{Mg}$ & $19.9 \pm 2.0$ & $17.3 \pm 1.9$ & $17.0 \pm 2.8$ & $102 \pm 10$ & $92 \pm 11$ & $104 \pm 14$ \\
\hline $\mathrm{Mn}$ & $52.0 \pm 2.8$ & $35.6 \pm 3.5$ & $48.5 \pm 3.6$ & $7.4 \pm 0.9$ & $7.7 \pm 1.0$ & $7.2 \pm 0.9$ \\
\hline $\mathrm{Ni}$ & $52.8 \pm 3.2$ & $49.3 \pm 2.9$ & $54.6 \pm 3.6$ & $43.4 \pm 3.5$ & $28.8 \pm 2.5$ & $32.7 \pm 3.7$ \\
\hline $\mathrm{Ti}$ & $118 \pm 14$ & $79.3 \pm 8.9$ & $111 \pm 19$ & $246 \pm 25$ & $124 \pm 13$ & $214 \pm 26$ \\
\hline V & $76.2 \pm 8.6$ & $52.2 \pm 5.4$ & $71.0 \pm 8.5$ & $30.1 \pm 2.0$ & $18.3 \pm 2.0$ & $32.0 \pm 4.3$ \\
\hline
\end{tabular}

Note: mean value \pm 3 S.D $(n=5)$.

better than all mentioned methods owing to the extremely low blanks, large applicable sample portions, high transport efficiency and the addition of Freon 1,2.

\subsection{Analysis of SiC samples}

The results obtained using slurry nebulization and using the solution nebulization for the analysis of various particle size silicon carbide powders were listed in Tables 3 and 4. For sub$\mu \mathrm{m}$ size of SiC powder, the results from slurry nebulization ICP-OES were calibrated using aqueous standards. However, for the $\mu \mathrm{m}$ size of SiC powder slurry nebulization ICP-OES, the results were calibrated only by using Certified Reference Material SiC slurries.

\section{Conclusion}

A simple, rapid and liable method was developed for the determination of trace impurities in high purity silicon carbide. The stable and homogeneous slurry should be prepared by addition of $0.5 \mathrm{wt} \%$ PEI as dispersant. The proposed method is suitable for various particle sizes of $\mathrm{SiC}$ powder. Owing to a rather low contamination in the sample preparation, the LODs, which are in the range of 40-2000 $\mathrm{ng} \mathrm{g}^{-1}$, superior to those of the conventional nebulization technique by ICP-OES or ICP-MS.

\section{Acknowledgments}

The authors are grateful to Shanghai Committee of Science and Technology (0159nm075) and Shanghai Institute of Ceramics, CAS (ZRJJ 200401) for financial support.

\section{References}

[1] K. Niihara, J. Ceram. Soc. Jpn. 99 (1991) 974.

[2] Y. Sakka, D.D. Bidinger, I.A. Aksay, J. Am. Ceram. Soc. 78 (1995) 479.

[3] T. Graule, A.V. Bohlen, J.A.C. Broekaert, E. Grallath, R. Kloekenkamper, P. Tschopel, G. Tolg, Fresenius J. Anal. Chem. 335 (1989) 637.

[4] B. Docekal, J.A.C. Broekaert, T. Graule, P. Tschopel, G. Tolg, Fresenius J. Anal. Chem. 342 (1992) 113

[5] J.A.C. Broekaert, R. Brandt, F. Leis, C. Pilger, D. Pollmann, P. Tschopel, G. Tolg, J. Anal. At. Spectrom. 9 (1994) 1063.

[6] M.T. Larrea, I.G. Pinilla, J.C. Farinas, J. Anal. At. Spectrom. 12 (1997) 1323.

[7] J.A.C. Broekaert, C. Lathen, R. Brandt, C. Pilger, D. Pollmann, P. Tschopel, G. Tolg, Fresenius J. Anal. Chem. 349 (1994) 20.

[8] U. Schaffer, V. Krivan, Anal. Chem. 71 (1999) 849.

[9] P. Barth, V. Krivan, J. Anal. At. Spectrom. 9 (1994) 773.

[10] Z. Wang, Z.M. Ni, D.R. Qiu, T.Y. Chen, G.Y. Tao, P.Y. Yang, J. Anal. At. Spectrom. 19 (2004) 273

[11] Z. Wang, Z.M. Ni, D.R. Qiu, T.Y. Chen, G.Y. Tao, P.Y. Yang, Winter Conference on Plasma Spectrochemistry, Fort Lauderdale, Florida, TP13, 2004.

[12] J.M. Mermet, J. Anal. At. Spectrom. 20 (2005) 11.

[13] A. Fisher, M.W. Hinds, S.N. Nelms, D.M. Penny, P. Goodall, J. Anal. At. Spectrom. 17 (2002) 1624

[14] I. Varga, F. Csempesz, G. Zaray, Spectrochim. Acta Part B 51 (1996) 253.

[15] J.C. Farinas, R. Moreno, J.M. Mermet, J. Anal. At. Spectrom. 9 (1994) 841

[16] L. Ebdon, M. Foulkes, K. Sutton, J. Anal. At. Spectrom. 12 (1997) 213

[17] J. Sun, L. Gao, J. Eur. Ceram. Soc. 21 (2001) 2447.

[18] L. Ebdon, M. Foulkes, S. Hill, J. Anal. At. Spectrom. 5 (1990) 67.

[19] M. Franek, V. Krivan, Fresenius J. Anal. Chem. 342 (1992) 118.

[20] B. Docekal, V. Krivan, J. Anal. At. Spectrom. 7 (1992) 521. 